Abstract

\title{
Focusing on the Chemical Characterization, Antioxidant and Cytotoxic Properties of Two Geophytes: Crocus Pallasii and Cyclamen Cilicium ${ }^{+}$
}

\author{
Gokhan Zengin ${ }^{1, *}$ and Evren Yildiztugay ${ }^{2}$ \\ 1 Department of Biology, Science Faculty, Selcuk University, 42130 Konya, Turkey \\ 2 Department of Biotechnology, Science Faculty, Selcuk University, 42130 Konya, Turkey; \\ eytugay@gmail.com \\ * Correspondence: gokhanzengin@selcuk.edu.tr; Tel.: +90-332232781 \\ + Presented at the 3rd International conference on Natural Products for Cancer Prevention and Therapy, \\ Kayseri, Turkey, 18-20 December 2019.
}

Published: 25 December 2019

\begin{abstract}
Species of the Crocus and Cyclamen genus have been reported to possess diverse biological properties. In the current work, the antioxidant and cytotoxic effects of the methanolic extracts of $C$. pallasii and C. cilicium aerial and underground parts were evaluated. The flower extracts of $C$. pallasii and $C$. cilicium possessed highest flavonoid content. Highest phenolic content was recorded from $C$. cilicium root extract $(47.62 \mathrm{mg}$ gallic acid equivalent/g extract). C. cilicium root extract showed significantly $(p<0.05)$ high radical scavenging $(94.28$ and $139.60 \mathrm{mg}$ trolox equivalent [TE]/g extract, against DPPH and ABTS radicals, respectively) and reducing potential (173.30 and $109.53 \mathrm{mg}$ TE/g extract, against CUPRAC and FRAP, respectively). Methanolic extracts of $C$. pallasii and C. cilicium showed toxicity against breast cancer cell lines. In the light of the above findings, C. cilicium might be considered as an interesting candidate in the development of anti-cancer agent, possessing antioxidant properties.
\end{abstract}

Keywords: Crocus; Cyclamen; antioxidant; cell assay; natural agents

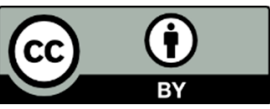

(C) 2019 by the authors. Licensee MDPI, Basel, Switzerland. This article is an open access article distributed under the terms and conditions of the Creative Commons Attribution (CC BY) license (http://creativecommons.org/licenses/by/4.0/). 